Case Report

\title{
Large Intra-Osseous Cystic Meningioma in a Child: A Rare Entity
}

\author{
Denlewende Sylvain Zabsonre ${ }^{1,}$, , Ben-Aziz Dao ${ }^{1}$, Valentin Konsegre ${ }^{2}$, Eric $\mathrm{Nao}^{3}$, Yacouba Haro ${ }^{1}$, \\ Ido Fabrice $^{2}$, Yves Bako ${ }^{4}$, Boureima Kinda ${ }^{4}$, Abel Kabre ${ }^{1}$ \\ ${ }^{1}$ Neurosurgery Department of Yalgado Ouedraogo Teaching Hospital, Joseph KI-ZERBO University, Ouagadougou, Burkina Faso \\ ${ }^{2}$ Pathology Anatomy Department of Yalgado Ouedraogo Teaching Hospital, Joseph KI-ZERBO University, Ouagadougou, Burkina Faso \\ ${ }^{3}$ ORL Department of Yalgado Ouedraogo Teaching Hospital, Joseph KI-ZERBO University, Ouagadougou, Burkina Faso \\ ${ }^{4}$ Anesthésia and Reanimation Department of Yalgado Ouedraogo Teaching Hospital, Joseph KI-ZERBO University, Ouagadougou, Burkina \\ Faso
}

\section{Email address:}

szabsonre@gmail.com (Denlewende S. Z.), benazizd@yahoo.fr(Ben-Aziz D.), vallentin9@gmail.com (Valentin K.), martialnao@yahoo.fr(Eric N.), yaksterharo@gmail.com (Yacouba H.), idojfrabrice@yahoo.fr(Ido F.), yvesbako@yahoo.fr(Yves B.), kindab2@yahoo.fr (Boureima K.), kabrel@yahoo.fr(Abel K.)

${ }^{*}$ Corresponding author

\section{To cite this article:}

Denlewende Sylvain Zabsonre, Ben-Aziz Dao, Valentin Konsegre, Eric Nao, Yacouba Haro, Fabrice Ido, Yves Bako, Boureima Kinda, Abel Kabre. Large Intra-Osseous Cystic Meningioma in a Child: A Rare Entity. Clinical Neurology and Neuroscience. Vol. 3, No. 2, 2019 , pp. 31-34. doi: 10.11648/j.cnn.20190302.11

Received: April 8, 2019; Accepted: May 21, 2019; Published: June 11, 2019

\begin{abstract}
Introduction. Meningiomas are rare tumors in children, in whom the intracranial pathology is dominated by craniopharyngiomas and medulloblastomas. Cystic meningiomas are also rare, as is their intraosseous location. Intraosseous meningiomas are ectopic meningiomas. Their diagnosis is rarely done preoperatively. We report a case of cystic intraosseous meningioma managed in our department. Observation. Mr. ON, 16 years old, presented progressively a right temporal tumefaction causing an aesthetic prejudice. This tumefaction raised the flag of the right ear. It was covered by normal skin and there was moderate right hypo acoustics. CT scan showed a right sphenotemporal lesion with hyperdense contours and hypodense contents. It repressed the brain without invading it but affected the anatomic structures of the ear. Surgical procedure allowed for almost complete excision of the lesion. Histology of the operative specimen concluded to an intraosseous meningothelial meningioma. After a 4-year follow-up, the tumor residue remained stable. Conclusion. In addition to the diagnostic difficulty, we faced two other therapeutic difficulties. The first is that complete excision was not possible to avoid compromising a hearing always good. The second difficulty was related to the age of the patient who did not allow us to consider the cranial plasty before the end of the growth.
\end{abstract}

Keywords: Intraosseous Meningioma, Partial Resection, Hearing

\section{Introduction}

Meningiomas are rare tumors in children in whom the intracranial tumor pathology is dominated by craniopharyngiomas and medulloblastomas [1]. Cystic meningiomas are also rare [2] as is their location in intraosseous [3]. Intraosseous meningiomas are ectopic meningiomas. According to some authors [4], Hoye classifies these ectopic meningiomas into four types. Type A corresponds to the externalization of an initially intracranial meningioma. Type B refers to meningiomas that arise from extracranial arachnoid cells, located along the fusion zones of the embryonic skull and spine, or along the sheaths of the cranial nerves. Type $\mathrm{C}$ corresponds to the ectopic primitive 
meningioma with no relation to the cranial nerves. Type D of Hoye corresponds to metastases of an intracranial malignant meningioma. The diagnosis of intraosseous meningiomas is rarely done preoperatively. We have found in the literature some cases of intraosseous meningioma in mass. However, we found no case of intraosseous meningioma in the cystic form. We report a case of intraosseous, cystic meningioma managed in the neurosurgery department of Yalgado Ouedraogo Teaching Hospital of Ouagadougou from January 2012 to November 2015.

\section{Case Report}

Mr. ON, a 16-year-old student, farmer's son, was referred to our department by a general surgeon from his province for the management of a cranial swelling.

$\mathrm{Mr} \mathrm{ON}$ did not report any pathological antecedent. He presented a right temporal swelling of slow and progressive increase in 6 years and causing an aesthetic prejudice (disfigurement) for which the patient consulted

Examination noted a hard, painless right temporal swelling, fixed in the deep plane, oval, about $8 \mathrm{~cm}$ long, raising the flag of the right ear and covered with a healthy skin.

CT scan showed spontaneous hypodensity (cyst) intraosseous right temporosphenoidal limited by bone hyperdensity. These lesions do not take the contrast. They are well limited and push back the brain without invading it but touched the bone structures of the right ear. In bone window, the scanner revealed an appearance of hyperostosis and osteolytic in places (figure 1).

An ORL examination (audiometry) asked for the impact of the structures of the ear on the CT scan concluded to a minimal right hypoacusis

Double-team surgical procedure (neurosurgeon, ORL) allowed partial resection of the lesion in mass to avoid compromising the hearing of the patient who was still good. A more or less clear liquid flowed at the opening of the tumor mass. The dura under the lesion was uninvested but very thin, letting out cerebrospinal fluid. The closure was made without a cranioplasty leaving a bone void (covered by the temporal muscle) created by the removal of the lesion.

Pathologic anatomy of the operative specimen found intraosseous meningothelial meningioma with no atypical features (figure 2).

The postoperative course was simple. A subcutaneous collection of cerebrospinal fluid was effectively treated with iterative punctures and a compression bandage. The patient was discharged from hospital on the fifth postoperative day. He was seen in consultation at 1 month, 3 months, 6 months and every year for 4 years before being lost sight. He did not report any complaints as much on his hearing as on the aesthetic. Clinically, the lesion residue remained stable (Figure 3). No control imagery could be performed for lack of financial means. In the same way, a possible bone plasty is still waiting.

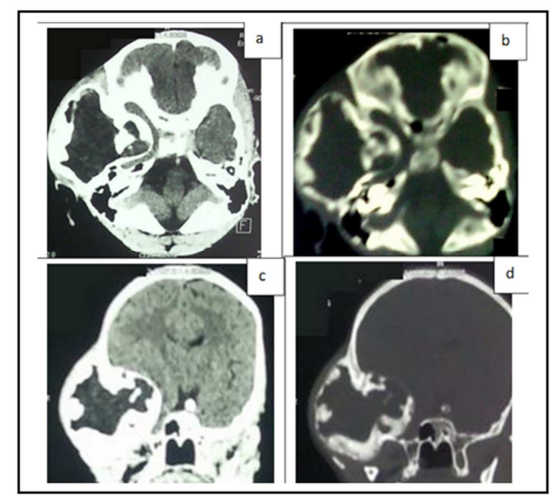

Figure 1. CT scan. Preoperative CT scan after contrast product injection in axial section parenchymal window (a); bone window (b); sagittal reconstruction parenchymal window (c); bone window (d). It show right intraosseous hypodensity temporosphenoidal limited by bone hyperdensity. These lesions are not take contrast. They repressed the brain without invading it but affected the structures of the. In bone window there is an aspect of hyperostosis osteolytic in places.

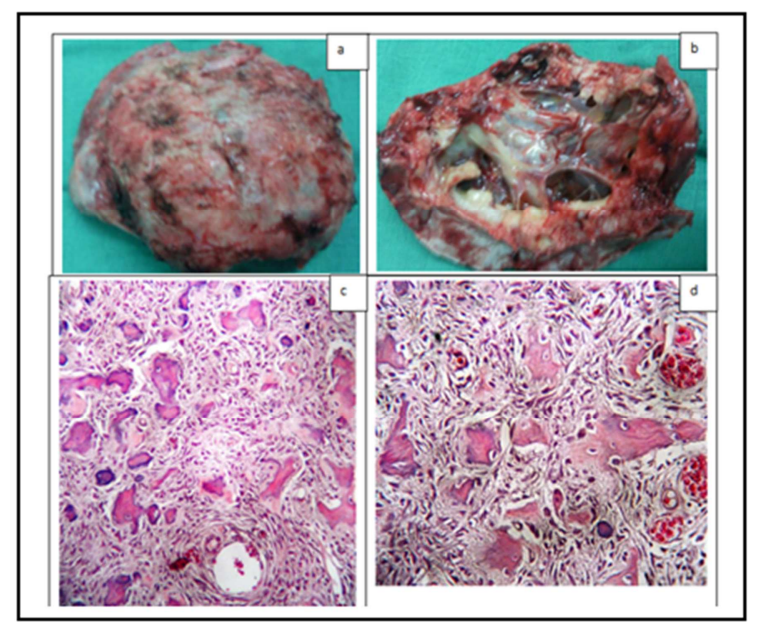

Figure 2. Pathologic anatomy. Macroscopy: Exocranial view (a); endocranial (b) of the operative specimen. Surgical piece of $12 \times 8 \times 5 \mathrm{~cm}$ wrapped by a bone shell and revealing at its cut soft tissue of whitish fibrous appearance with micro calcifications adhering firmly to the bone. Microscopy (photo taken with digital camera directly at the microscope eyepiece) $H E$ (eosin hematic) coloration $20 \times 10$ (c) and 40x 10 (d) magnification field. Proliferation of meningothelial cells of lobular architecture, interspersed with Haversian bone fragments and numerous calcospherites. These meningothelial cells of syncytial appearance are regular without atypia. Nuclei are round or oval with fine chromatin. There are some winding images "whorls". There is no necrosis or mitosis. There are sometimes congestive blood vessels with clean walls.

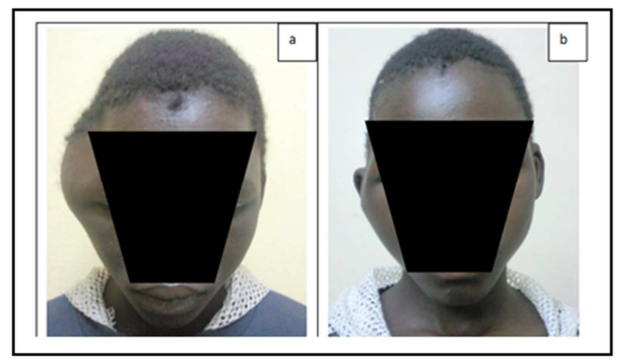

Figure 3. Clinical aspects. Right temporal tumefaction preoperatively (a) and at 6 months postoperatively (b). An almost total disappearance of the swelling is observed, of which a small part (left in place to preserve the hearing) persists in pre-auricular 


\section{Discussion}

Primary interosseous meningiomas are rare among ectopic meningiomas. Most authors have described unique cases of intraosseous hyperostosis meningiomas [3-5]. We did not find any cystic form of interosseous meningiomas occurring in a child like ours. Cystic meningiomas are rare with difficult diagnosis [2]. Similarly, brain tumors are rare in children. Medulloblastomas and craniopharyngiomas are the most common brain tumors in children. One case of intra-parenchymal ectopic meningioma has been described [1] Primary interosseous meningiomas develop from intraosseous meningeal cells. It may be either multipotent mesenchymal cells that differentiate into meningocytes; or sequestration of meningocytes during suture fusion during intra-uterine development or during head injury with fracture [6-8].

We did not find a notion of trauma in our case. The neurological examination of the patient was normal. He consulted for aesthetic prejudice (disfigurement) caused by the swelling. Authors have also found a normal neurological examination [4-6]. The discrete hypo-acoustics have been accidentally diagnosed with audiometry for the impact of the structures of the ear on the CT scan. The existence of neurological signs could be related to a large intracranial extension of the tumor causing nerve compression [6,9]. Exophthalmia and / or visual troubles related to spheno temporo-orbital location of intraosseous meningiomas were the most noted physical signs in the literature $[5,7,9]$

Authors [2-5] agree on the polymorphic and non-specific aspect of the lesion on imaging. Thus two forms have been described: the form called hyperostosis is the most common, and the osteolytic or erosive form. Intra-tumor calcification may occur in $20 \%$ of cases [3, 4]. The osteolytic form has a more aggressive evolution or represent a later form of the disease [3]. The CT scan allows to see in addition to bone lesions, the meningioma which has a tissue density [4] and / or cystic [2] as it was our case. Tissue lesions may enhance after injection of contrast product [4]. Similarly, it will be necessary to seek a contrast enhancement of the dura under the lesion [3, 5]. No lesional enhancement is visible in condensed forms [5]. No enhancement was noted in our case because it was a cystic and form surrounded by a condensed form. Magnetic resonance imaging allows a better analysis of the underlying dura and intra cranial extensions of the lesion [3]. The injection of gadolinium causes contrast enhancement within the intraosseous component and at the level of the dura under [4, 5]. Indeed, some authors may have an underlying lesion of dura, while for others it is another entity called meningiomas in plate [3-5].

We did not mention the diagnosis of intraosseous meningioma before histology. This diagnosis is rarely mentioned in front of clinic and imaging. In general the aspects evoke a benign or malignant bone tumor. The diagnosis of certainty of intraosseous meningioma is a histological surprise. Low grade meningiomas are by far the most common [4, 6, 9]. A case of intraosseous meningioma cause of false positives in the search for bone metastases by bisphosphonate scintigraphy has been described [10].
Surgical resection should be as complete as possible, taking all the bone involved. A bone reconstruction could be considered immediately or in a second time $[6,8,11]$. An extemporaneous histology often helps to take the decision to make or not this plasty at the same time operative. Radiation therapy should be considered in cases of high grade meningioma or partial excision of low grade [9].

\section{Conclusion}

The diagnosis of intraosseous meningioma was postoperative by histology when it had not even been mentioned preoperatively. In addition to this diagnostic difficulty, we were confronted with two other therapeutic difficulties. The first is that despite a double team surgery (neurosurgeon and ORL), complete excision was not possible to avoid compromising an always good hearing on the side of the lesion. However, the tumor residue remained stable for many years. The second difficulty was related to the patient's age, which was that of a child still growing. This did not allow us to consider cranial plasty before the end of this growth.

\section{References}

[1] Khursheed N, Rumana M, Rayees M, Altaf R. Intraparenchymal anaplastic meningioma in a child: A rare entity. asianjns. org; 2015, DOI: 10. 4103/1793-5482. 154980, 10(2): $1-3$

[2] Souei Mhiri M, Ben Rhouma K, Tlili-graiess K, El Ouni Salhi C, Jemni Gharbi H, Ben Hnia I, Krifa H, Mokni M. Aspects en imagerie par résonance magnétique des méningiomes kystiques à propos de quatre cas. Neuroradiol., 2005, 32, 54-58.

[3] Benchakrouna F, Ech-cherif El Kettani N, Arkhab Y, Chakir N, El Khamlichi A, El Hassani MR, Jiddane M. Le méningiome intra-osseux sphéno temporo-orbitaire. Feuillets de radiologie 2011; 51: 254-259.

[4] Elkharras A, En-nouali H, Jawhari N, Elhaddad A, Ajja A, Chaouir S, Benamaur M. Méningiome géant a développement intra et extracranien. AJNS 2005; 24 (1)

[5] Henon A, Colombat M, Rodallec M, Redondo A, Feydy A. Méningiome intra-osseux de la voûte du crâne: confrontation anatomo-radiologique. JR 2005; 01: 86-1

[6] El Mahou S, Popa L, Constantin A, Jamard B, Cantagrel A, Mazieres B, Laroche M. Multiples intraosseous meningiomas. Clin Rheumatol, 2006, 25: 553-554. 6

[7] Nasri S, Marzouki Zineb, Kamaoui Imane, Skiker1 Imane, Le méningiome intra osseux: à propos de deux cas. Pan African Medical Journal. 2018; 30: 220. doi:10. 11604/

[8] Zhang S, Zhang Jing, Chen J, Ai X, He X. Frontal intradiploic meningioma with progressive intracranial invasion. A rare case report. Medicine, 2017; 96:34.

[9] Luetjens G, Krauss JK, Brandis A, Nakamura M. Bilateral sphenoorbital hyperostotic meningiomas with proptosis and visual impairment:A therapeutic challenge. Report of three patients and review of the literature. Clinical Neurology and Neurosurgery, 2011; 113: 859-863. 
[10] Ridouh M, Megueni AZ, Krim M, Mahida B, Berber N. Meningiome intra-osseux:cause de faux positifs dans la recherche de metastases osseuses par scintigraphie aux biphosphonates. revue neurologique, 2015; 171: 122-127.
[11] Broeckx CE, Maal TJJ, Vreeken RD, Bos RRM, Ter Laan M, Single-step resection of an intraosseous meningioma and cranial reconstruction: technical note World Neurosurgery, 2017; DOI: 10. 1016. 